\title{
Notional number agreement in English
}

\author{
KARIN R. HUMPHREYS and KATHRYN BOCK \\ University of Illinois at Urbana-Champaign, Urbana, Illinois
}

\begin{abstract}
To investigate the contested role of notional number in English subject-verb agreement, we used a sentence completion task to examine agreement with minimally different subject noun-phrases, such as the gang on the motorcycles and the gang near the motorcycles. These contrasting phrases biased different notional construals of collective nouns, such as gang, which are normally ambiguous between plural (distributed) and singular (collected) construals. With subjects biased toward spatial distribution, such as gang on motorcycles, more plural verbs occurred in speakers' sentence completions than in sentence completions with a bias toward spatial collection, such as gang near motorcycles. This offers strong evidence regarding both the existence and the magnitude of notional effects on subjectverb number agreement in English.
\end{abstract}

The traditional description of English subject-verb agreement is that verbs agree in number with the subjects of their clauses. Less clear is whether verbs agree with the notional or grammatical number of the subject, or both. Notional number is the numerosity of the subject's referent in the speaker's mental model, and grammatical number is the conventional linguistic number of the subject (head) noun. Examples of both kinds of agreement exist. For nouns like scissors, verb agreement seems to be grammatical (The scissors were/*was sharp); the word draws a plural verb despite the singularity of the referent. In other cases, agreement seems notional. Morgan (1972) gave the example bacon and eggs tastes good, in which singular tastes agrees not with the phrase's plural grammatical number, but with a notional construal of bacon and eggs as a set or name.

Controlled studies have investigated the balance between notional and grammatical agreement in English by providing subjects for speakers to complete as sentences and then noting the verb number used in the completions. These studies have yielded mixed effects. Bock and Miller (1991), replicated by Vigliocco, Butterworth, and Garrett (1996), found no effect of notional plurality when distributivity was manipulated, comparing distributive subjects like the label on the wine bottles with nondistributives like the key to the cabinets. Although both phrases

Portions of this work were presented at the University of Southern California Language Production Conference, Los Angeles, November 1999. The first author was supported by a National Science Foundation graduate fellowship and a training grant from the National Institutes of Health (T32 MH 19990). Funding for the research came in part from grants from the National Science Foundation (SBR 94-11627, SBR 9873450, BCS-0214270) and the National Institutes of Health (R01 HD21011, R01 MH66089). We thank Erica Middleton, Matthew Rambert, Lisa Octigan, Angela Swendsen, Betsy Taylor, Seema Patel, and Rebecca Barrick for assistance in collecting data and Eric Solomon for providing his rating instructions. Correspondence should be addressed to K. R. Humphreys at the Beckman Institute, University of Illinois, 405 N. Mathews Avenue, Urbana, IL 61801 (e-mail: jkbock@uiuc.edu). are grammatically singular, the former is normatively taken to refer to multiple tokens of one type and the latter to a single token. In contrast, strong effects of distributivity have been found in Dutch, French, Spanish, and Italian (see Vigliocco, Butterworth, \& Garrett, 1996; Vigliocco, Butterworth, \& Semenza, 1995; Vigliocco, Hartsuiker, Jarema, \& Kolk, 1996), inviting the hypothesis that the sparseness and simplicity of English agreement morphology make it comparatively insensitive to notional number (Vigliocco, Hartsuiker, et al., 1996).

At odds with this conclusion is a finding by Eberhard (1999) that notional number, when made more salient, does affect English verb agreement. Also challenging a purely grammatical account of English verb agreement is the fact that English speakers seem alert to the notional plurality latent in collective nouns (e.g., gang). With collective-noun subjects, American English speakers used plural verbs at a rate intermediate between the rates for regular singular and regular plural subjects (Bock, Nicol, \& Cutting, 1999), suggesting some role for notional number.

Despite these findings, the effect of notional number on agreement remains unclear. Existing studies have employed materials that confounded notional number variations with other properties, thus making the actual notional effect uncertain. Completely different subject noun-phrases are used, as in the label and key examples above, with the notional contrast established intuitively or by norming (see Bock \& Miller, 1991; Eberhard, 1999; Vigliocco, Butterworth, \& Garrett, 1996; Vigliocco et al., 1995; Vigliocco, Hartsuiker, et al., 1996). In the best controlled test (Bock et al.,1999), the heads of subject noun phrases varied between collective and noncollective nouns. Because individual nouns, even nouns with similar meanings, vary enormously in agreement properties (compare cattle are and stock is), the absence of control over these properties fuels the debate about whether, how, and to what extent notional number influences agreement. 
The aim of the work reported here was to assess the impact of distributivity in minimally contrastive contexts. We exploited a property of collective nouns that stems from their ambiguity in number reference. The ambiguity arises because groups can be regarded as units or as multitudes of individual members: One can see either the forest $o r$ the trees. We manipulated notional number by creating phrases in which the referents of collective nouns, which served as the heads of subject phrases, were likely to be construed as more or less spatially distributed. Consider the gang on the motorcycles and the gang near the motorcycles. A gang on motorcycles is separated across space in a way that a gang near motorcycles need not be. We assumed that spatial separation made individual members more salient, whereas spatial collection made the group more salient, in accordance with basic Gestalt properties (Wertheimer, 1923). Variation along this dimension encourages representations that are notionally more or less plural.

In our experiment, notional number was manipulated by changing only the preposition (e.g., on vs. near) in a modifier. This kept the major lexical and grammatical properties of the subjects identical, while changing the notional bias from plural to singular. If verb number covaries with notional number when grammatical number remains constant, we would argue that notional number must play a role in English verb agreement.

The phrases were presented to speakers who repeated and completed them as sentences. The speakers used the phrases as sentence subjects and added predicates to complete the sentence. We expected notionally plural phrases to elicit plural verbs. However, a competing source of plural number is attraction. Attraction occurs when a verb agrees with a plural noun in the modifier of a singular subject, as in the time for fun and games are over (Bock $\&$ Miller, 1991). The contribution of attraction to notional agreement in previous research is hard to estimate for the same reasons that the notional contribution is moot. To estimate better the strength of attraction, we included control preambles with singular local nouns (e.g., the gang near the motorcycle).

The baseline for plural agreement was provided by preambles with grammatically plural heads (e.g., delinquents replaced gang). This baseline also served to assess whether differences between alternative construals of the collectives could be due to differences in prepositions rather than to differences in distributivity. That is, if the relation signaled by near tends to trigger singular agreement and that signaled by on tends to trigger plural agreement, for example, similar variations should be in evidence for the plural heads.

Finally, we manipulated the modality of presentation. In research using visual presentation, notional effects have tended to be strong, whereas in research using auditory presentation, the notional effects have tended to be weak (excepting Eberhard, 1999, and Vigliocco et al., 1995, Experiment 1). If notional variations are comprehended better when preambles are read than when they are heard, or if written presentation exacerbates the complexity of distributive construals and increases variability in agreement, visual presentation should elicit more plural agreement than should spoken presentation in the distributive condition.

If notional number influences subject-verb agreement generally, the use of plural verbs should be more widespread after distributively biased preambles (e.g., the gang on the motorcycles) than after collectively biased preambles (the gang near the motorcycles). The increase in plural verbs should occur over and above any increase attributable to plural attraction, as estimated from differences in plural agreement after singular and after plural local nouns. Alternatively, if notional number has no reliable effect on English subject-verb agreement, plural agreement should be equivalent for distributed and collected construals, with equivalent amounts of attraction.

\section{METHOD}

\section{Participants}

The 144 participants were native English-speaking undergraduates at the University of Illinois. For their participation, they received either payment or class credit.

\section{Materials}

The experimental preambles were noun phrases with a head noun and a prepositional phrase modifier (e.g., the gang on the motorcycles). There were 18 experimental items. Each item appeared in six conditions (see Table 1). The head noun was either collective or plural (e.g., gang or delinquents). The local noun in the prepositional phrase was singular (motorcycle) or plural (motorcycles). Prepositions were varied to create either a distributed or a collected construal of the head noun (e.g., on or near); for consistency, we designated the prepositional phrases in the plural head-noun conditions in the same way. There were 15 different preposition pairs. To avoid peculiar phrases like the gang on the motorcycle, distributive prepositions never accompanied singular local nouns.

Table 1

Example Preambles for One Item

\begin{tabular}{llll}
\hline \multicolumn{1}{c}{ Preamble } & Head Noun & Local Noun & Construal \\
\hline The gang on the motorcycles & collective & plural & distributed \\
The gang near the motorcycles & collective & plural & collected \\
The gang near the motorcycle & collective & singular & collected \\
The delinquents on the motorcycles & plural & plural & distributed \\
The delinquents near the motorcycles & plural & plural & collected \\
The delinquents near the motorcycle & plural & singular & collected \\
\hline
\end{tabular}


We created six lists. Each included one representative of every experimental item, three in each of the six experimental conditions. Interspersed with the experimental items were 50 fillers. Fillers had regular singular or plural head nouns that, when there were multiple nouns, matched in number. All experimental items are listed in the Appendix.

\section{Procedure}

Half the participants read the preambles, and half heard recordings of them made by a female speaker of American English. Participants were tested individually using a Macintosh Quadra 650 with a 17-in. monitor running PsyScope experimental software (Cohen, MacWhinney, Flatt, \& Provost, 1993). Responses were recorded onto digital audio or audiocassette tapes.

On every trial, participants viewed a cross in the center of the screen for $800 \mathrm{msec}$, followed by presentation of the preamble. Auditory preambles were presented over external speakers; visual preambles appeared on the monitor. Visual display time was adjusted for preamble length by allocating a constant $250 \mathrm{msec}$ plus $25 \mathrm{msec}$ for each function word character and $40 \mathrm{msec}$ for each content word character, giving most participants just enough time to read the preamble once. Preamble offset was followed immediately by an exclamation point to cue the response. Participants then repeated and completed the preamble.

\section{Design}

Presentation modality was a between-subjects and within-items variable, with 72 participants and all 18 items in each cell. All other variables were within participants and within items. These were head noun (collective or plural), local noun (plural or singular), and construal (distributed or collected). Head- and local-noun types were crossed, and construal was nested within the combination of head-noun type with plural local nouns. In each of the resulting six conditions, every participant received three items, and every item was presented to 24 participants.

\section{Scoring}

Responses were transcribed and coded for verb number. Number was coded as singular, plural, uninflected, or miscellaneous. Singular or plural responses occurred when the preamble was repeated accurately and an unambiguously number-inflected verb was produced. When speakers self-corrected, we coded only the first verb uttered. Responses were classified as uninflected when the preamble was repeated accurately but number was indeterminate. This occurred chiefly when past tense regular verbs were produced (e.g., walked). Finally, miscellaneous responses occurred when pream- bles were incorrect, unintelligible, or unusually disfluent; when they were not the sentence subject; or when the completion contained no intelligible verb.

\section{RESULTS}

Table 2 shows the distribution of responses. Generally, auditory presentation elicited more number-inflected responses and fewer miscellaneous responses than did visual presentation. In analyses of variance with head, local number/construal, and modality as factors, auditory presentation produced significantly more plural responses $\left[F_{1}(1,142)=9.3\right.$ and $F_{2}(1,17)=16.8$ by participants and items, respectively] and significantly fewer miscellaneous responses $\left[F_{1}(1,142)=118.7\right.$, and $\left.F_{2}(1,17)=50.4\right]$. However, because the major effects involving distributivity were the same regardless of presentation (all $F \mathrm{~s}<1$ ), we collapsed the remaining results over modality except when significant modality differences occurred. The dependent variable was the percentage of plural verbs out of all responses. Critical analyses were conducted using planned $t$ tests, one set with participants $\left(t_{1}\right)$ and another with items $\left(t_{2}\right)$ as random variables. Unless otherwise indicated, contrasts were significant at or below the .05 level.

Figure 1 gives the proportions of plural verbs among the number-inflected responses. Collective heads were uniformly less likely than plural heads to take plural verbs, .57 to .99 . These proportions represent $19.2 \%$ and $32.4 \%$ of all responses produced.

The crucial comparison in this study was between the distributed and collected construals of preambles with collective heads and with plural local nouns (the gang on the motorcycles vs. the gang near the motorcycles). Significantly more plural verbs accompanied distributed than accompanied collected construals, $27.5 \%$ to $19.9 \%$ $\left[t_{1}(143)=2.83\right.$, and $\left.t_{2}(17)=3.11\right]$ corresponding to .74 and .67 of the number-inflected responses, respectively. When head and local nouns were both plural, plural verbs constituted .97 or more of the inflected responses in all

Table 2

Numbers of Responses by Condition, Classification, and Modality of Presentation

\begin{tabular}{|c|c|c|c|c|c|c|c|}
\hline \multicolumn{3}{|c|}{ Preamble Condition } & \multirow[b]{2}{*}{ Modality } & \multicolumn{4}{|c|}{ Response Classification } \\
\hline Head Noun & Local Noun & Construal & & Plural & Singular & Uninflected & Miscellaneous \\
\hline \multirow[t]{2}{*}{ collective } & plural & distributed & visual & 48 & 19 & 98 & 51 \\
\hline & & & auditory & 72 & 22 & 108 & 14 \\
\hline \multirow[t]{2}{*}{ collective } & plural & collected & visual & 36 & 16 & 93 & 71 \\
\hline & & & auditory & 56 & 29 & 105 & 26 \\
\hline \multirow[t]{2}{*}{ collective } & singular & collected & visual & 18 & 35 & 102 & 61 \\
\hline & & & auditory & 21 & 65 & 112 & 18 \\
\hline \multirow[t]{2}{*}{ plural } & plural & distributed & visual & 69 & 1 & 93 & 53 \\
\hline & & & auditory & 75 & 0 & 128 & 13 \\
\hline \multirow[t]{2}{*}{ plural } & plural & collected & visual & 63 & 0 & 86 & 67 \\
\hline & & & auditory & 85 & 0 & 105 & 26 \\
\hline \multirow[t]{2}{*}{ plural } & singular & collected & visual & 48 & 3 & 89 & 76 \\
\hline & & & auditory & 83 & 1 & 115 & 17 \\
\hline
\end{tabular}




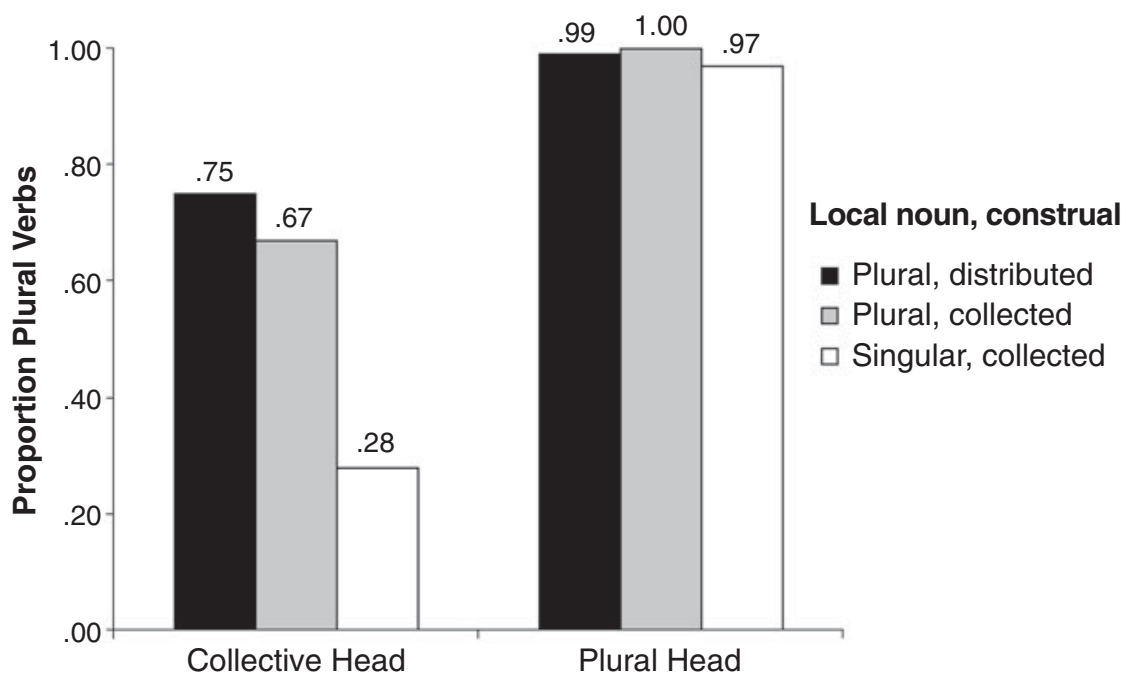

Figure 1. Overall proportion of plural verbs among inflected responses.

conditions, yielding no differences between the distributed and collected construals (all $t \mathrm{~s}<1$ ). To test the interaction between head noun and construal when the local noun was plural, we performed analyses of variance with head noun (collective vs. plural) and construal (collected vs. distributed) as factors. Singular local-noun conditions were excluded, because their construal did not vary. The interaction was significant $\left[F_{1}(1,143)=\right.$ $\left.5.03 ; F_{2}(1,17)=4.85\right]$, indicating that construal affected collective but not plural heads.

The other important comparison gauged the role of attraction and compared plural agreement after both singular and plural local nouns. This was done only for collective heads with a collected construal (the gang near the motorcycles vs. the gang near the motorcycle). There were significantly more plural verbs after plural local nouns than there were after singular local nouns, $19.9 \%$ to $10.2 \%\left[t_{1}(143)=3.89\right.$, and $\left.t_{2}(17)=4.50\right]$, equaling .67 and .28 of the inflected verbs. This difference was significant in both modalities but was greater for auditory presentation than for visual presentation $\left[F_{1}(1,142)=\right.$ $\left.7.01 ; F_{2}(1,17)=3.69, p=.07\right]$.

The same pattern of results was obtained in analyses performed on the arcsin-transformed proportions of plurals (calculated relative to all number-inflected responses in each condition). These analyses were done for items only, because of the large number of empty cells for individual subjects.

\section{DISCUSSION}

More plural verbs occurred with distributed than with collected construals. That is, the notional number of subject phrases had small but reliable effects on verb agreement when their grammatical properties remained constant. This adds a crucial piece of evidence to previous findings of notional verb agreement in English. Other studies that have varied notional number have done so by varying the entire phrase or the type of head noun, leaving open the possibility that what were interpreted as notional effects were actually effects of other variables. Among the uncontrolled variables were (1) the plausibility of materials in different conditions (distributive construals are sometimes less sensible), (2) differences in comprehensibility, (3) differences in the grammatical properties of component words, and so on. Only the contributions of imageability to distributivity have been carefully addressed: Eberhard (1999) found that highly imageable subject phrases made notional number agreement more likely. But even that is contested. Reviewing Eberhard's (1999) account of her findings, Vigliocco and Hartsuiker (2002, p. 455) conjectured that imageability affects the incidence of agreement errors rather than agreement itself, the implication being that notional number is not at work in ordinary English agreement except as a distraction.

Because the differences we obtained occurred within closely matched distributive and nondistributive contexts, with controls for the effects of attraction, there is less room to argue about the underlying nature of notional number agreement in English. Our results implicate distributivity itself as a force behind the use of plural verb number in English, rather than attractions or distractions from imageability.

The possibility nonetheless remains that factors stemming from the preposition variation contributed to our results. To examine this more closely, we gathered ratings of our items on two other notional dimensions that have been implicated in agreement. The first dimension was imageability (Eberhard, 1999). Following Eberhard's instructions, 20 raters judged the imageability of the phrases with collective heads and with plural local 
nouns, receiving either the collected or the distributed versions on one of two counterbalanced lists. Imageability was rated on a scale of 1 (low) to 7 (high). The second dimension was semantic integration (Solomon \& Pearlmutter, 2004). In spoken sentence-completion tasks, Solomon and Pearlmutter found that semantic integration, a sort of conceptual coherence, increased attraction in subject-verb agreement. For example, subject nounphrases such as the drawing of the flowers were associated with more attraction than phrases such as the drawing with the flowers. Their interpretation was that more semantic integration allows more simultaneity in production planning, increasing the occurrence of attraction. To assess whether this influenced our results, we also collected judgments of semantic integration from another group of 20 raters, using the same lists employed for the imageability ratings.

Table 3 gives the mean item ratings and their correlations with the proportions of plural agreement. Distributed construals were rated as significantly more imageable than were collected construals (by a Wilcoxon signed-ranks test, $Z=2.48$ ), in keeping with the supposition that distributivity increases the salience of individuals. However, the contribution of imageability to plural agreement was negligible, as is indicated by the near-zero correlation. The rated degree of semantic integration was significantly larger for collected than for distributed construals $(Z=3.72)$, but again, this had little influence on plural agreement. Notably, the direction of the ratings is the reverse of what would be required for semantic integration to account for our results.

In contrast, when collectivity and distributivity were treated as a two-valued variable, the correlation with plural agreement was significant $(r=.37)$. Partialing out imageability and semantic integration changes this correlation very little, though it becomes slightly stronger $(r=.39)$. Obviously, this does not mean that imageability and semantic integration play no role in agreement. Studies by Eberhard (1999) and by Solomon and Pearlmutter (2004) have indicated that they do play a role, in one case, as a modulating factor (distributive construals are more salient when situations are more concrete), and in the other, as an effect on attraction. The finding does mean that neither imageability nor semantic integration made significant contributions to our results. We cannot categorically rule out other confounds with the preposition manipulation, but we believe that the results are

Table 3

Mean Ratings of Preambles With Collected and Distributed Construals on Imageability and Semantic Integration and Correlations of Ratings With Plural Verb Agreement

Mean Ratings

\begin{tabular}{lcc}
\multicolumn{1}{c}{ Construal } & Imageability & Semantic Integration \\
\cline { 2 - 3 } Collected & 5.1 & 4.07 \\
Distributed & 5.5 & 3.21 \\
Correlation with proportion & & \\
$\quad$ of plural agreement & $r=.11$ & $r=-.09$ \\
\hline
\end{tabular}

more plausibly attributed to distributivity than to systematic lexical properties of the 15 different preposition pairs used over the 18 experimental items.

The mechanism behind notional effects on verb agreement, as well as the process by which the verb comes to bear the number of the subject, remains in dispute. Although both our findings and those of Eberhard (1999) imply that the mechanism need not differ across languages, there are attractive reasons to believe that it may. Vigliocco, Butterworth, and Garrett (1996) proposed that in Italian and Spanish, the verb takes its number directly from the message-level representation, rather than from the subject noun-phrase (as proposed for English and Dutch by Bock, Eberhard, Cutting, Meyer, \& Schriefers, 2001). In most instances, the two mechanisms yield the same result, but direct marking allows notional number to be reflected unambiguously on verbs regardless of whether or not grammatical and notional number are consistent in the subject noun phrase.

Direct marking is nonetheless difficult to resolve with the weakness of notional agreement in English and with findings from speech errors. When nouns erroneously move into subject position, verbs almost invariably agree with the spurious subject. Stemberger (1985) reported that verbs agreed with spurious subjects in six of the seven relevant exchange errors in his corpus, as in most cities are true of that (produced instead of that is true of most cities). Stemberger found a similar pattern in the four relevant noncontextual errors in his corpus: In your teeth are all red, a statement in which the singular tongue was the intended subject, substitution of the plural teeth elicited a correspondingly plural verb. These kinds of errors are typically characterized by a mismatch between the speaker's intention and the meaning conveyed by the actual utterance. Unless exchanges and word substitutions give rise to revisions of notional number, the errors are explained better by mechanisms that allow verbs to take their number from the subject, even when the wrong noun phrase is placed in the subject position.

The results of the present study help to clarify another wellspring of controversy about distributivity in English. The matching of our distributive and nondistributive materials allows a better estimate of the impact of notional number on agreement when the lexical properties of the subject are controlled. With well-matched preambles, we found an increase of less than $8 \%$ in plural agreement. This effect is miniscule in comparison with that created by variations in the grammatical number properties of head nouns, and it is about the same size as the effect of attraction, which is also small. When an effect of this subtlety is sought amid other variations in agreement properties, it is easily obscured.

This does not mean that distributivity is inconsequential. When different sources of notional plurality are combined, the effects of distributivity can be substantial. We found a strong tendency toward plural agreement for collective heads with plural local nouns (replicating Bock et al., 1999). The increase in plurality was approx- 
imately $36 \%$ higher after plural local than after singular local. This is much larger than the attraction effect typically seen with noncollective singular heads, where the differences between singular and plural local nouns range between 5\% and 15\% (Bock \& Eberhard, 1993; Bock \& Miller, 1991; Bock et al., 1999). One explanation for the magnitude of this effect involves notional influences (Bock et al., 1999). In comparison with the singular local nouns, plural locals may predispose a more distributed construal of the subject. That is, a gang near motorcycles is more likely to be distributed around them than is a gang near a single motorcycle. In short, putting a group into a distributive situation signaled by a plural modifying noun can magnify its notional plurality.

The weakness of differences due to presentation modality suggests that this factor has little effect on agreement performance. The larger number of miscellaneous responses that occurred with visual presentation (as in Bock \& Eberhard, 1993, Experiments 1 and 2), however, indicates that input factors such as comprehensibility or short-term memory may be more likely to compromise production after visual than after auditory presentation, at least in the current implementation.

Our finding of notional number sensitivity suggests that English subject-verb agreement is not entirely unlike that in Dutch, Italian, French, and Spanish. There may be cross-language differences in notional agreement along the lines observed by Vigliocco and colleagues (Vigliocco, Butterworth, \& Garrett, 1996; Vigliocco et al., 1995), but the differences are likely to be graded rather than sharp. Even within languages, notional number affects agreement in variable ways across experimental stimuli and situations: In English, our results and those of Eberhard (1999) suggested notional effects, whereas Bock and Miller's (1991) and Vigliocco, Butterworth, and Garrett's (1996) findings suggested none; in Spanish, Vigliocco, Butterworth, and Garrett's (1996) findings suggested notional effects, whereas those of Carreiras and Meseguer (2001), using a slightly different methodology, suggested none.

In conclusion, it appears that subtle variations in the notional number of sentence subjects can affect verb agreement in English. This finding implies that the im- plementation of verb number agreement is influenced not only by the grammatical number properties of subject nouns but also by the number properties of the mental referents of subject noun-phrases.

\section{REFERENCES}

Bock, K., \& Eberhard, K. M. (1993). Meaning, sound and syntax in English number agreement. Language \& Cognitive Processes, 8, 57-99.

Bock, K., Eberhard, K., Cutting, J. C., Meyer, A. S., \& Schriefers, H. (2001). Some attractions of verb agreement. Cognitive Psychology, 43, 83-128.

Bock, K., \& Miller, C. A. (1991). Broken agreement. Cognitive Psychology, 23, 45-93.

Bock, K., Nicol, J., \& Cutting, J. C. (1999). The ties that bind: Creating number agreement in speech. Journal of Memory \& Language, 40, 330-346.

CARreIras, M., \& Meseguer, E. (2001, September). Gender and number agreement in sentence comprehension and production in Spanish. Paper presented at the meeting of the European Society for Cognitive Psychology, Edinburgh.

Cohen, J. D., MacWhinney, B., Flatt, M., \& Provost, J. (1993). PsyScope: An interactive graphic system for designing and controlling experiments in the psychology laboratory using Macintosh computers. Behavior Research Methods, Instruments, \& Computers, 25, 257-271.

EBERHARD, K. M. (1999). The accessibility of conceptual number to the processes of subject-verb agreement in English. Journal of Memory \& Language, 41, 560-578.

Morgan, J. L. (1972). Verb agreement as a rule of English. In P. M. Peranteau, J. N. Levi, \& G. C. Phares (Eds.), Papers from the Eighth Regional Meeting, Chicago Linguistic Society (pp. 278-286). Chicago: Chicago Linguistic Society.

Solomon, E. S., \& Pearlmutter, N. J. (2004). Semantic integration and syntactic planning in language production. Cognitive Psychology, 49, 1-46.

STEMBERGER, J. P. (1985). An interactive activation model of language production. In A. W. Ellis (Ed.), Progress in the psychology of language (Vol. 1, pp. 143-186). London: Erlbaum.

Vigliocco, G., Butterworth, B., \& Garrett, M. F. (1996). Subjectverb agreement in Spanish and English: Differences in the role of conceptual constraints. Cognition, 61, 261-298.

Vigliocco, G., Butterworth, B., \& Semenza, C. (1995). Constructing subject-verb agreement in speech: The role of semantic and morphological factors. Journal of Memory \& Language, 34, 186-215.

Vigliocco, G., \& HARTSUIKER, R. J. (2002). The interplay of meaning, sound, and syntax in sentence production. Psychological Bulletin, 128, 442-472.

Vigliocco, G., Hartsuiker, R. J., Jarema, G., \& Kolk, H. H. J. (1996). One or more labels on the bottles? Notional concord in Dutch and French. Language \& Cognitive Processes, 11, 407-442.

WERTHEIMER, M. (1923). Untersuchungen zur Lehre von der Gestalt, II. Psychologische Forschung, 4, 301-350. 


\section{APPENDIX \\ Experimental Preambles}

1. The crowd/commuters inside/outside the subway car/s

2. The jury/jurors in/near the folding chair/s

3 . The crew/sailors in/for the lifeboat/s

4. The clan/princes in/with the ancient castle/s

5. The gang/delinquents on/near the motorcycle/s

6 . The hired help/servants in/from the mansion/s

7. The squad/soldiers inside/behind the armored vehicle/s

8. The team/drivers in/close to the race car/s

9. The secretarial staff/secretaries in/around the tiny cubicle/s

10. The family/sisters on/with the new bicycle/s

11. The wedding party/celebrities in/beside the stretch limousine/s

12. The group/friends on/next to the jet ski/s

13. The boy scout troop/boy scouts inside/outside the tent/s

14. The orchestra/musicians on/by the tour bus/es

15. The audience/spectators in/beneath the luxury box/es

16. The class/students at/near the lunch table/s

17. The tribe/natives inside/outside the stone hut/s

18. The posse/cowboys on/near the horse/s

Note-Items 4, 7, 10,11, 13, and 17 in the visual condition used the determiner their instead of the in the prepositional phrase, which may have predisposed the verbs in responses to be plural. However, this predisposition would have been constant across construal conditions.

(Manuscript received April 30, 2002;

revision accepted for publication February 5, 2003.) 